\title{
Evaluating outcomes after cardiac surgery: A complex challenge requiring flexibility
}

\author{
Filippo Rapetto, MD, and Vito Domenico Bruno, MD, $\mathrm{PhD}$
}

\author{
From the Bristol Heart Institute, University of Bristol, School of Clinical Sciences, Bristol, United Kingdom. \\ Disclosures: Authors have nothing to disclose with regard to commercial support. \\ Received for publication May 31, 2018; revisions received May 31, 2018; accepted for publication June 1, 2018; \\ available ahead of print July 14, 2018. \\ Address for reprints: Vito Domenico Bruno, MD, PhD, School of Clinical Science, University of Bristol, Research \\ Floor Level 7, Bristol Royal Infirmary, Upper Maudlin St, BS2 8HW Bristol, United Kingdom (E-mail: Vito.D. \\ Bruno@bristol.ac.uk). \\ J Thorac Cardiovasc Surg 2018;156:1916-7 \\ $0022-5223 / \$ 36.00$ \\ Copyright (c) 2018 by The American Association for Thoracic Surgery \\ https://doi.org/10.1016/j.jtcvs.2018.06.015
}

In the present issue of the Journal, Diab and colleagues ${ }^{1}$ present their institutional outcomes for patients undergoing cardiac surgery according to different intensive care unit lengths of stay. In a prospective single-center cohort study, the authors focused their analysis on longitudinal assessment of patients' quality of life and quality of recovery.

The article inspires some comments about the current era of cardiac surgery and its future directions; more specifically, it raises questions about the way the cardiac surgery community reports its results and, ultimately, assesses itself.

Historically, we have been trained to report our outcomes mainly in terms of survival and early postoperative complications rates. Considering the consistent and significant decrease in mortality and morbidity, it is becoming more and more evident that a wider perspective needs to be embraced. $^{2,3}$ Reporting that our patients survive to the operations is not enough anymore; we need to demonstrate how they survive and what their quality of life looks like as the time goes by. It is also important to highlight that although it is sometimes useful and certainly appealing to conceptualize health as a dichotomous and absolute entity, it is clear that the actual scenario is far more complex: Time-dependence and clustering are frequently involved in modern analysis. Moreover, health markers do not change suddenly and irreversibly, but vary over time in different directions, and the variables of interest are not always measured at the same time intervals for each individual.

Quality of life and quality of recovery perfectly exemplify our statements: They are useful variables for analyzing clinical outcomes, and there is growing evidence demonstrating their validity and reproducibility in cardiac surgery; they are nondichotomous variables that can be tested multiple times for each subject and can improve or worsen at every timepoint.

Unfortunately, although these are surely simple concepts from a theoretical point of view, introducing such variables

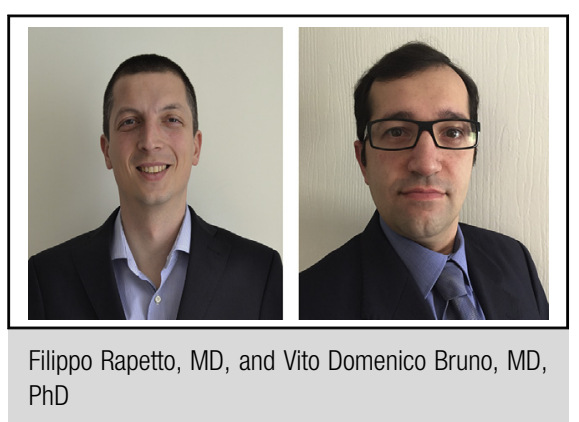

Central Message

Quality of life and quality of recovery are emerging measures of outcome in cardiac surgery. Longitudinal analysis and linear mixed models are precious tools to incorporate into statistical analysis.

See Article page 1906. into statistical models adds significant complexity to the analysis.

In their article, Diab and colleagues ${ }^{1}$ used the PostopQRS tool (Dataphiles Ltd, Otley, UK) to measure a patient's quality of recovery at 7 different moments, from the preoperative period to 12 months postoperatively. The aim of their analysis was to detect differences in quality of recovery over the study period in 2 groups of patients defined by different intensive care unit length of stay. To conduct the study, the authors needed a model capable of incorporating different levels of information, namely, accounting for variability between groups while adjusting for longitudinal variability caused by multiple repeated measurements. ${ }^{4}$ In such a scenario, flexibility of the model is the key. Linear mixed model allowed to highlight not only a different overall recovery at 12 months between the 2 groups but also, more important, a different pattern of recovery over time even when similar values were found at the end of the study.

In terms of clinical outcomes, Diab and colleagues ${ }^{1}$ have supported their analysis using propensity score (PS) matching; the presence of differences in preoperative characteristics could have had an impact on the clinical outcomes, but the authors avoided this risk by using PS matching, thus eliminating any further doubts on their results. We have been aware of the benefits of PS matching for a long time, ${ }^{5}$ and it is not the aim of this article to renew these 
concepts, but it is important to highlight the flexible statistical thinking behind this study and the benefits derived from it.

This article well exemplifies how current perspectives in cardiac surgery demand more complex statistical analysis to move on from traditional modeling strategies. A flexible and effective statistical strategy is essential when reporting our long-term results and will become even more important in the future of cardiac surgery clinical research.

\section{References}

1. Diab MS, Bilkhu R, Soppa G, Edsell M, Fletcher N, Heiberg J, et al. The influence of prolonged intensive care stay on quality of life, recovery, and clinical outcomes following cardiac surgery: a prospective cohort study. J Thorac Cardiovasc Surg. 2018; $156: 1906-15$.

2. Myles PS, Hunt JO, Fletcher H, Solly R, Woodward D, Kelly S. Relation between quality of recovery in hospital and quality of life at 3 months after cardiac surgery. Anesthesiology. 2001;95:862-7.

3. Eagle KA, Guyton RA, Davidoff R, Edwards FH, Ewy GA, Gardner TJ, et al. ACC/AHA 2004 guideline update for coronary artery bypass graft surgery: a report of the American College of Cardiology/American Heart Association task force on practice guidelines (committee to update the 1999 guidelines for coronary artery bypass graft surgery). Circulation. 2004; 110:e340-437.

4. Andrinopoulou E-R, Rizopoulos D, Jin R, Bogers AJJC, Lesaffre E, Takkenberg JJM. An introduction to mixed models and joint modeling: analysis of valve function over time. Ann Thorac Surg. 2012;93:1765-72.

5. Blackstone EH. Comparing apple and oranges. J Thorac Cardiovasc Surg. 2002; $123: 8-15$. 